\title{
Schizophrenia and NICE: all quiet on the community front?
}

\section{Mark Salter}

\author{
"No battle plan survives contact with \\ the enemy" attributed to Sun Tzu
}

Although the concept of psychotic illness was known to the healers of his age, Sun Tzu did not have mental illness in mind when he penned those words two and a half thousand years ago. His maxim nonetheless remains true of both our responses to war and to schizophrenia. They have much in common. Both are universally associated with images of horror and darkness. Both carry an awful cost-1\% of the human population will suffer a psychotic breakdown, often in young adulthood. Both are ever changing. War has altered beyond recognition since 1911 when Bleuler first coined the term "schizophrenia" to describe a shattering of the mind. When the Captains of American psychiatry met last year to consider their future nomenclatures, they came close to dropping the $\mathrm{S}$ word altogether. Over here, our top brass - their chateaus as far from the front line as ever-are talking instead of "salience disorder".

Many psychiatrists, accustomed to the realities of care for people with psychotic illness in a world of broken families and broken windows, will at once be struck by the discrepancy between the order and optimism enshrined in the recently revised National Institute for Health and Clinical Excellence (NICE) guidelines for schizophrenia ${ }^{1}$ and the stark reality of the world seen in their ward rounds and clinics. It is a document for our times, a battle plan driven by a rationalist philosophy that places great faith in objectivity. Those who write the history of Western thought in years from now will probably see the oxymoron more clearly than we do.

Clues that this faith in objectivity may be misplaced, however, are not hard to find. The introduction to the guidance reminds us that the sheer variety of symptoms embraced by the term schizophrenia may well represent a "cluster of disorders". Any notion that the differences between such putative clusters might crucially inform

Correspondence to: Dr Mark Salter, Adult General and Community Psychiatrist, East London Foundation NHS Trust, City and Hackney Centre for Mental Health, London E9 65R, UK; mark.salter@eastlondon.nhs.uk assessment, diagnosis and management of the condition, however, is lacking. Further on, we are reminded that schizophrenia is often comorbid with other conditions, such as post-traumatic stress disorder, personality disorder and substance misuse. The possibility that these conditions may comprise the core of the condition-and the evidence base for such a notion is substantial $^{2}$ - receives no consideration. The stigmatisation of the condition is acknowledged too, yet this concept informs little of the guidance in the pages that follow, beyond a need to acknowledge cultural differences. After saluting truths that battle hardened clinicians have known for decades, the guidance trundles on like a tank over the trenches, its gunfire indifferent to the sophistication of its enemy.

Mental health workers have long grown used to the ceaseless bombardment of guidelines, protocols and inquiry reports that nowadays steer the path of service design. The lexicon of truisms will at once be familiar to veteran readers of this document, with its reminders of the need for "person-centred care" or the fact that "good communication between health care professionals and service users is essential". What is commendable about the document is the height at which it sets its sights. It is explicit about the fact that all individuals found to be suffering with schizophrenia, regardless of severity or duration of illness, should routinely be offered psychosocial interventions alongside drug oriented treatments. Adequate physical health care is essential. Iatrogenic side effects must always be monitored. It is explicit about what these interventions should comprise. But the distance between chateau and trench remains visible; the guidance advises specific therapies for the management of negative syndromes but does not dwell on the stark fact that negative features in their many forms comprise the bulk of our work. Elsewhere, it skips lightly over the seams in a seamless service-few of our patients are ever likely to receive continuous psychological care from the same worker as they pass through the bewildering maze that nowadays comprises our service.

Few clinicians would disagree that The National Service Framework for mental health set out after 1997 led to a welcome improvement in services for people with schizophrenia after decades of neglect. Whether the framework of "functional teams" within which clinicians were obliged to deliver it was necessarily the best way to do so is less certain. The forces behind this implementation can hardly be said to reflect the principles of evidence based practice that this guidance purports to embrace. In the 1990s there was no real evidence base to support the superiority of Early Intervention, Assertive Outreach and other elite squadrons over the hardy troopers of generic Community Mental Health Teams. The cultural backdrop to the reprovision too was far from calm and rational; risk and lurid reportage of serious untoward incidents dominated public and government thinking for much of its gestation.

Privately, many frontline clinicians feel that like so many government initiatives spawned at that time, it was driven less by carefully garnered wisdom than by charismatic individuals with something new to say that chimed with the optimism of the time. The message that has since emerged is more sobering ${ }^{3}$ but this intelligence is only slowly filtering back to $\mathrm{HO}$. In the field, the modus operandi of these crack troops is less distinct than our leaders might hope. What is the real difference between a diligent generic Community Mental Health Team worker caring for someone with a chronic psychotic illness compared with her counterpart in a specialist rehabilitation team? Target populations are no less harder to define in the fog of work. Assertive Outreach teams are clogging with patients who seem too unwell, or too risky, to return to generic teams, let alone primary care.

This guidance pinpoints another aspect of practice camouflaged by our claims of objectivity; our preoccupation with the notion of the expert as opposed to the generalist. It enshrines the notion that best practice involves moving patients around a network of specialists, each skilled to meet the varying, unique needs identified by the "person-centred" process. Anyone who has cared for someone with schizophrenia in the real world knows that what works best is a stable relationship with an understanding, patient and skilled person who stays with the patient, and their illness, over time, through thick and thin. Just how to achieve this in a culture hell bent on atomisation requires careful thought. One solution might involve the creation of an entirely new breed of well paid, well 
trained community care workers: part therapist, part housing officer, part prescriber. Just what such a real world mental health trooper might look like has been described in detail. ${ }^{4}$ The battles that they face will be remote from the world depicted in the latest NICE guidance.

Competing interests: None.

1. National Institute for Health and Clinical Excellence. Core interventions in the treatment and management of schizophrenia in primary and secondary care (update). Clinical Guidelines CG82, issued March 2009. http://www.nice.org.uk/guidance/ index.jsp?action $=$ bylD\&o $=11786$ (accessed 29 July 2009).
2. Kendell R. The distinction between personality disorder and mental illness. Br J Psychiatry 2002;180:110-15.

3. Killaspy $\mathbf{H}$, Bebbington $\mathrm{P}$, Blizard $\mathrm{R}$, et al. The REACT study: randomised evaluation of assertive community treatment in north London. BMJ 2006;332:815-20.

4. Salter M, Turner T. Community mental health care: a practical guide to outdoor psychiatry. Edinburgh: Churchill Livingstone, 2008.

\section{Are all antidepressants equal?}

\section{G Gartlehner, ${ }^{1,2}$ B N Gaynes ${ }^{3}$}

Primary care physicians and psychiatrists manage the majority of patients suffering from acute phase major depressive disorder (MDD). For most patients, antidepressant treatment is the primary choice of care. Second generation antidepressants (SGAs)-developed following the first generation of tricyclic and monoamine oxidase agents-have become the preferred drug choice because of their greater tolerability, lower risk of lethality and similar efficacy compared with first generation agents.

Clinicians prescribing SGAs face a multitude of drug choices and are the target of extensive marketing campaigns by the pharmaceutical industry. In 2007, three of the 20 top selling drugs in the USA were antidepressants with annual sales ranging from $\$ 2.3$ billion (venlafaxine XR (Effexor XR)) to $\$ 1.4$ billion (duloxetine (Cymbalta)). ${ }^{1}$ At the time of writing, 13 different SGAs have been approved for the treatment of major depression in the USA and Canada and two additional drugs (reboxetine, milnacipran) are available in some European countries. Some of these drugs are now available as generic medications, others are still patent protected. Economically, drug choice matters. The US Consumers Union found that in 2008 the average monthly costs of treatment with second generation antidepressants in the USA varied from $\$ 20$ to $\$ 400$ depending on the medication of choice. ${ }^{2}$ The study

\footnotetext{
${ }^{1}$ Danube University, Department for Evidence-based Medicine and Clinical Epidemiology, Krems, Austria; ${ }^{2}$ Sheps Center for Health Services Research, University of North Carolina at Chapel Hill, Chapel Hill, USA; ${ }^{3}$ Department of Psychiatry, School of Medicine, University of North Carolina at Chapel Hill, Chapel Hill, USA

Correspondence to: Dr G Gartlehner, Danube University, Department for Evidence-based Medicine and Clinical Epidemiology, Krems, Austria; gerald. gartlehner@donau-uni.ac.at
}

conducted by Cipriani and colleagues ${ }^{3}$ addressed an ongoing challenge for clinicians: how to choose among antidepressant treatments and select the best drug for an individual patient suffering from an episode of major depression (see page 107).

\section{WHY SUCH A STUDY IS IMPORTANT}

The burning question for patients and clinicians is whether differences in costs are substantiated by differences in benefits and harms. Evidence based data to answer this question and to guide selection of treatments has been limited. Because of the lack of available direct head to head comparisons, prior systematic reviews have been able to say little about differences among the medications. $^{45}$ The ideal studies to fill this knowledge gap would be large, pragmatic, randomised controlled trials (RCTs) that directly compare the benefits and harms of SGAs. Unfortunately, out of the more than 70 possible comparisons among SGAs, barely more than half have been investigated in RCTs. ${ }^{6}$ Many of the available studies are small or have methodological problems that severely limit the ability to draw firm conclusions about the comparative efficacy and safety.

In a complex statistical analysis, Cipriani and colleagues ${ }^{3}$ compared response rates and discontinuation rates of individual SGAs and offered a simple answer: sertraline (Zoloft) and escitalopram (Lexapro) are better than other SGAs followed by venlafaxine (Effexor) and mirtazapine (Remeron). ${ }^{3}$ Is this the long sought for answer for clinicians who treat patients with MDD? An accompanying editorial in The Lancet "Antidepressants are not all created equal" was jubilant and asserted that "...a new gold standard of reliable information has been compiled..." Subsequent letters to the editor, however, were less enthusiastic but rather outright critical about the methods and conclusions of this study..$^{-13}$ The underlying tenor of the critics was that Cipriani et al failed to acknowledge the methodological limitations of the approach and, by ranking drugs, evoked an unsubstantiated sense of precision based on evidence that is fraught with uncertainty. A closer look at the underlying methods is necessary to understand the controversy.

\section{STUDY DESIGN AND METHODS}

The objective of the study was to provide a clinically useful summary of the effects of SGAs that can be used to guide treatment decisions. ${ }^{3}$ To detect relevant studies, authors conducted systematic literature searches in the Cochrane Collaboration depression, anxiety and neurosis trials register. Furthermore, they contacted pharmaceutical companies, regulatory agencies and study investigators to acquire unpublished or missing data. The primary outcomes were response (defined as a $50 \%$ improvement of the baseline score) and treatment discontinuation rates at 8 weeks.

Because not all antidepressants have been compared directly in RCTs, Cipriani and colleagues $^{3}$ used a statistical technique called multiple treatments metaanalysis to derive estimates of the comparative efficacy and safety for all possible comparisons among SGAs. Such an approach essentially combines results from trials directly comparing two or more SGAs with estimates of treatment effects based on common comparators. For example, if two drugs exhibit a similar treatment effect relative to a common comparator, the conclusion would be that these drugs have similar efficacy.

In the absence of direct comparisons, such an approach is legitimate and findings can allow inferences about the relative benefits (or harms) of drugs that have never been compared directly. Nevertheless, results have to be interpreted cautiously. Such a statistical approach is commonly viewed as observational evidence, even if the statistical 